\title{
INTERFACE CAPTURING IN DUAL-FLOW MICROFLUIDICS
}

\author{
Evgeniy Shapiro*, Dimitris Drikakis*, Joseph Gargiuli ${ }^{\dagger}$ and Pankaj Vadgama ${ }^{\dagger}$ \\ *Fluid Mechanics \& Computational Science Group, Aerospace Sciences Department, Cranfield \\ University, Cranfield, Bedfordshire, MK43 0AL, UK \\ e-mails: e.shapiro@cranfield.ac.uk, d.drikakis@cranfield.ac.uk \\ web page: http://www.cranfield.ac.uk/soe/fluid \\ ${ }^{\dagger}$ IRC in Biomedical Materials, Queen Mary, University of London, Mile End Road, London, E1 \\ $4 \mathrm{NS}, \mathrm{UK}$ \\ e-mails: j.f.gargiuli@qmul.ac.uk, p.vadgama@qmul.ac.uk
}

Key words: CFD, Microflows, Microfabrication

\begin{abstract}
Dual-fluid flows are utilised extensively in microfluidics applications. A problem of particular interest is the precise positioning of the contact discontinuity between the carrier fluids. The paper presents a computational study of the interface positioning in xylene/water flows used in polymer membrane fabrication in microfluidics. Both artificial compressibility, high-resolution characteristics-based and pressure-correction-based schemes have been employed. The former numerically captures the interface position, whereas the latter is used in conjunction with a volume-of-fluid interface tracking scheme. The high-resolution scheme results in less diffusive contact discontinuity and the predicted interface position compares favourably with analytic results.
\end{abstract}

\section{INTRODUCTION}

Utilisation of controlled dual-fluid laminar flows in microfluidic channels for microfabrication purposes was initially proposed by Kenis et al. ${ }^{1,2}$. The "Fabrication using Laminar flow" method (FLO) is based on the implementation of controlled reactions at the interface between laminar fluid streams. The main advantages of this approach include the possibility of limiting reactions to thin layers, control over the shape of the reaction zone via the control of the dual-fluid interface and possibility for in-situ fabrication inside MEMS devices.

Recently several attempts were made to apply this methodology for in-situ polymer $^{3,4,5,6}$ and biological ${ }^{7}$ membrane fabrication. For fast interfacial polycondensation reactions involved in nylon 6,6 membrane formation on the interface between an aqueous solution of hexamethylenediamine and adipoyl chloride, Gargiuli et al. ${ }^{6}$ demonstrated that the properties of the formed membrane can vary significantly depending on the geometry of the channel and local flow characteristics. Since the characteristic time for flow 
development in microfluidics is much less than the characteristic time of interfacial polycondensation $^{6}$, the position of the contact discontinuity can be determined by studying the flow of the carrier liquids alone.

An important effect, highlighted by Gargiuli et al. ${ }^{6}$ based on CFD studies of carrier liquids, is the displacement of the contact discontinuity between the carrier liquids due to the difference in carrier liquids viscosities pumped in with the same volume flux. The interface displacement in this flow occurs because the less viscous liquid (xylene) subjected to less friction moves faster thereby requiring smaller cross-section than the more viscous liquid (water) in order to generate the same volume flux. This effect was briefly discussed by Shapiro et al. ${ }^{8}$, and the interface position correction based on volume flux adjustment was later employed by Nair et al. ${ }^{7}$ to obtain the precise positioning of a biomembrane. Note that although the water/xylene flow with equal thickness layers is linearly unstable to both long and short wave disturbances ${ }^{9,10}$, these were not excited in polymer membrane fabrication experiments ${ }^{6}$ and stable interface between water and xylene was obtained. In computer simulations, numerical diffusion can lead to a more diffused displacement of the interface. This artificial diffusion is important in microflows for microfabrication purposes where the interface spreading can be of the same order of magnitude as the fabricated structures.

The aim of this paper is to present a comparative study of two different computational fluid dynamics methods for modelling the interface position: (i) a high-resolution characteristics-based scheme ${ }^{11,12}$ and (ii) a traditional pressure-correction based algorithms applied in conjunction with Volume Of Fluid (VOF) interface tracking ${ }^{13}$. For a two-dimensional flow setup, an analytic solution for the position of the interface is derived and used to assess the accuracy of the above schemes in the developed flow region. Moreover, we show that it is possible to re-position the interface in the middle of the microchannel using volume flux corrections obtained analytically. Finally, the accuracy of the results for computations performed on an interface-fitted grid is also discussed.

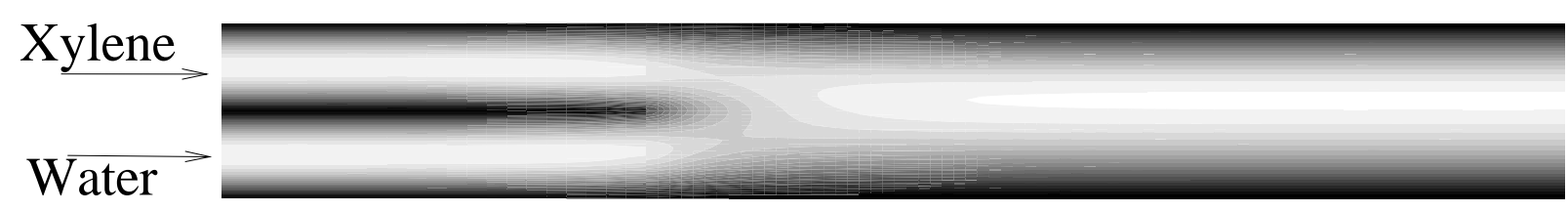

Figure 1: Microfluidic cell schematic including contours of streamwise velocity component) 
Table 1: Water/Xylene properties used in the present study.

\begin{tabular}{cll}
\hline Property & Water & Xylene \\
\hline$\rho, \frac{\mathrm{kg}}{\mathrm{m}^{3}}$ & 1000 & 860 \\
$\mu, \mathrm{ma} \cdot \mathrm{s}$ & 1.0 & $0.62-0.76$ \\
\hline
\end{tabular}

\section{PROBLEM FORMULATION}

We consider a dual-fluid xylene/water low Reynolds number flow in a microfluidic channel (Figure 1). The dimensions of the microfluidic channel were chosen similar to polymer membrane fabrication experiments ${ }^{6}$, i.e. the length of the inlet and main channel were chosen as $10 D$ and $20 D$, respectively, where $D$ is the inlet diameter. The properties of water and xylene are summarised in Table 1; two different viscosities of xylene were chosen to cover the range of isomers. All computations were performed at Reynolds number 10 , which corresponds to flow rates used previously for polymer membrane fabrication ${ }^{6}$. Note that for low speed flows, the entrance length does not vary significantly and the flow establishes within 2-4 channel diameters ${ }^{14,15}$, therefore the main section length of $20 D$ is sufficient.

The governing equations for multi-species flows based on the artificial compressibility formulation ${ }^{16}$ are given by

$$
\left\{\begin{array}{c}
\frac{\partial \vec{u}}{\partial \tau}+(\vec{u} \cdot \nabla) \vec{u}=-\frac{1}{\rho} \nabla p+\frac{1}{R e_{l}} \nabla^{2} \vec{u} \\
\frac{\partial p}{\partial \tau}+\beta \nabla \cdot \vec{u}=0 \\
\frac{\partial \rho}{\partial \tau}+(\vec{u} \cdot \nabla) \rho=0 \\
\frac{\partial \rho_{x}}{\partial \tau}+(\vec{u} \cdot \nabla) \rho_{x}=0 .
\end{array}\right.
$$

where $\tau$ denotes pseudo-time; $\rho$ and $\rho_{x}$ are the total density and density of xylene, respectively, and $\operatorname{Re}_{l}=\operatorname{Re}_{l}\left(\rho, \rho_{x}\right)$ is the local Reynolds number. The above system is solved using a high-resolution characteristic-based scheme (hereafter referred to as HR-CBS) developed for multi-component flows by Shapiro and Drikakis ${ }^{11,12}$.

The pressure-correction-based computations were performed using first and secondorder accurate upwind schemes in conjunction with the SIMPLE pressure-velocity coupling and Volume of Fluid interface tracking method (hereafter referred to as PC-VOF) ${ }^{13}$ as provided by the commercial code FLUENT (Fluent 6.2.16); the additional volumefraction equation was for xylene. The media properties (e.g. density and viscosity) in the Navier-Stokes equations were calculated by volume averaging based on the computed volume fraction of xylene. A parabolic velocity profile was set at the inlets and the flow variables were extrapolated at the outlet.

The computations were performed on a $45 \times 25$ grid in the inlets sections and a $149 \times 49$ grid in the main channel section. Doubling the grid size in each direction of the cross- 
section altered the velocity magnitude at the interface by $1.3 \%$. Validation of the CFD models used in this paper was reported previously by Shapiro and Drikakis ${ }^{11,12}$ for HRCBS and by Shapiro et al. ${ }^{8}$ for PC-VOF.

\section{ANALYTIC SOLUTION}

For a 2D microfluidic channel (large aspect ratio), the developed flow downstream of the inlet junction is a two-fluid Poiseuille flow. Let the channel boundaries be given by $y= \pm 1$ and the interface located at $H$. Let us also choose the transformed co-ordinate system $z=\frac{y-H}{1+H}$ (this transformation will considerably simplify the resulting expressions, then the flow field is governed by the following system of equations

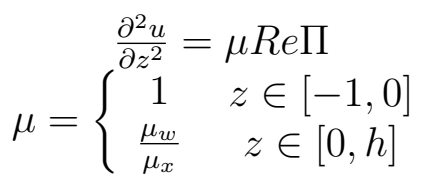

where $h=\frac{1-H}{1+H} \in(0,1]$ with $\mu_{x}$ and $\mu_{w}$ being the viscosities of xylene and water, respectively; $\Pi$ is the constant streamwise pressure gradient and Re denotes the Reynolds number based on water properties. The boundary conditions and volume conservation for 2 are given by

$$
\begin{gathered}
u(-1)=u(h)=0 \\
\mu_{w} \frac{\partial u}{\partial z}(-0)=\mu_{x} \frac{\partial u}{\partial z}(+0) \\
\int_{-1}^{0} u d z=Q_{w} \\
\int_{0}^{h} u d z=Q_{x}
\end{gathered}
$$

where $Q_{w}$ and $Q_{x}$ are the volume fluxes of water and xylene, respectively.

Solution of (2)-(3) for $\mu_{x w}=\frac{\mu_{x}}{\mu_{w}}, h$ and $Q_{x w}=\frac{Q_{x}}{Q_{w}}$ yields

$$
h^{4}+4 \mu_{x w} h^{3}+3 \mu_{x w}\left(1-Q_{x w}\right) h^{2}-4 Q_{x w} \mu_{x w} h-Q_{x w} \mu_{x w}^{2}=0
$$

On one hand for fixed media properties and volume fluxes, (4) allows to determine the interface displacement, $h$. On the other hand, if we would like to position the interface precisely in the centre of the channel, $h=1$, then (4) is reduced to

$$
\mu_{x w}^{2} Q_{x w}-7 \mu_{x w}\left(1-Q_{x w}\right)-1=0,
$$

which for given media properties allows to determine the corrected volume flux

$$
Q_{x w}=\frac{1}{\mu_{x w}} \frac{1+7 \mu_{x w}}{\mu_{x w}+7}
$$


a)

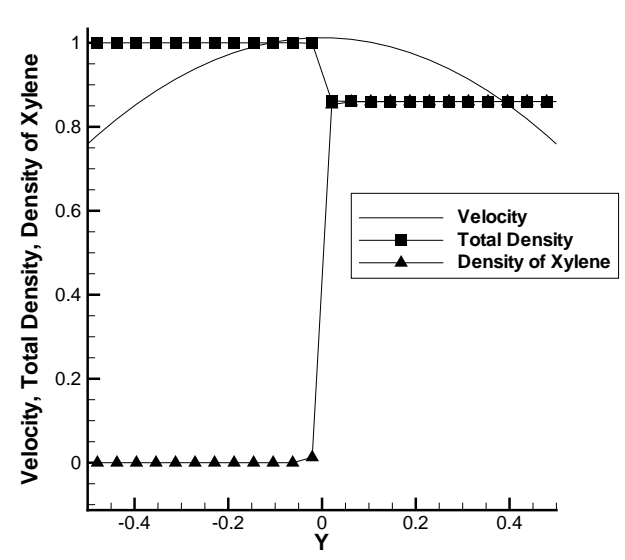

b)

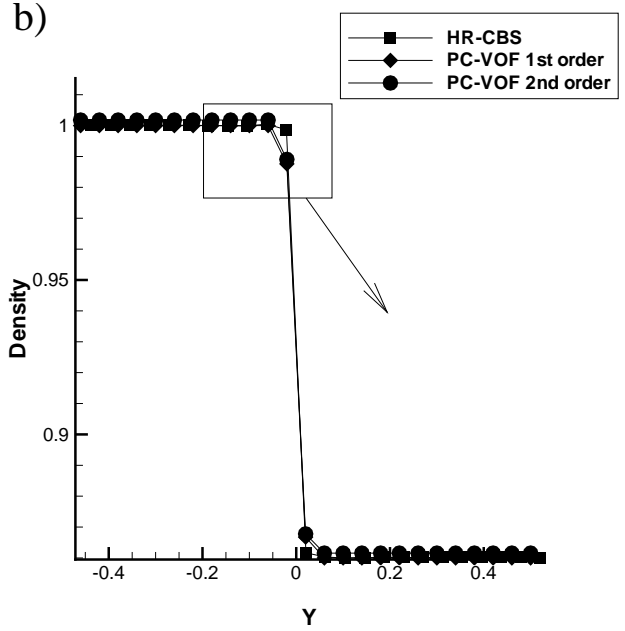

Figure 2: Contact discontinuity for equal viscosities: (a) solution obtained with HR-CBS; (b) density results across the interface using different computational models.

\section{RESULTS}

For viscosities ratio equal to 1 the contact discontinuity is aligned with the grid lines. Figure 2 shows the velocity, total density and xylene density across the channel section as obtained by using HR-CBS scheme, as well as comparisons of the results for the different computational models, i.e. HR-CBS, first and second order PC-VOF. HR-CBS yields sharper discontinuity than the PC-VOF.

For viscosity ratio different than one, the interface discontinuity is no longer aligned with the grid. Figures $3 \mathrm{a}$ and $3 \mathrm{c}$ show the solution (HR-CBS scheme) in the developed flow region for $\mu_{x w}=0.62$ and $\mu_{x w}=0.76$, respectively. The comparison of different models are presented in Figures $3 \mathrm{~b}$ and d. For viscosity ratio closer to one, the HR-CBS resolves the discontinuity within 3 points (one point inside the dicontinuity) and the PC-VOF within four points (two points inside the discontinuity). The (interpolated) position of the contact discontinuity (Table 2) as obtained by the HR-CBS model is slightly in better agreement with the analytic solution (Equation 4). It is worth noticing that although the flow is unstable according to linear theory ${ }^{9,10}$, instabilities at the interface of water/xylene flow were not observed in the polymer membrane fabrication experiments ${ }^{6}$ and other micro-fabrication processes ${ }^{1}$, where the viscosity of the liquid layers is different.

The spreading $5 \%$ of the contact discontinuity due to numerical diffusion might be considered acceptable in some practical applications, however, this is not negligible when precise positioning of the interface is required. For example, for the channels diameters used in microfabrication $(200 \mu \mathrm{m})^{1,2}, 5 \%$ spreading of the interface corresponds to $10 \mu \mathrm{m}$, which, in some cases, is comparable to the size of the fabricated structures.

The prediction of contact discontinuity can be improved using a re-mapped interfacefitted grid, which is applied to the initial solution obtained on a uniform grid. Taking 
a)

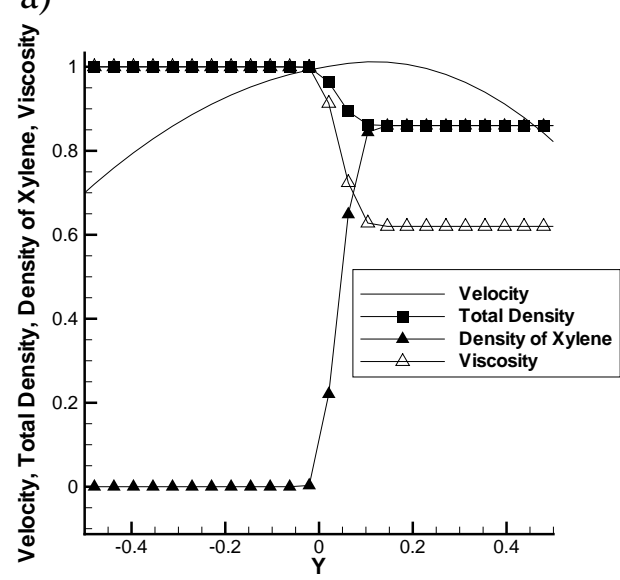

c)

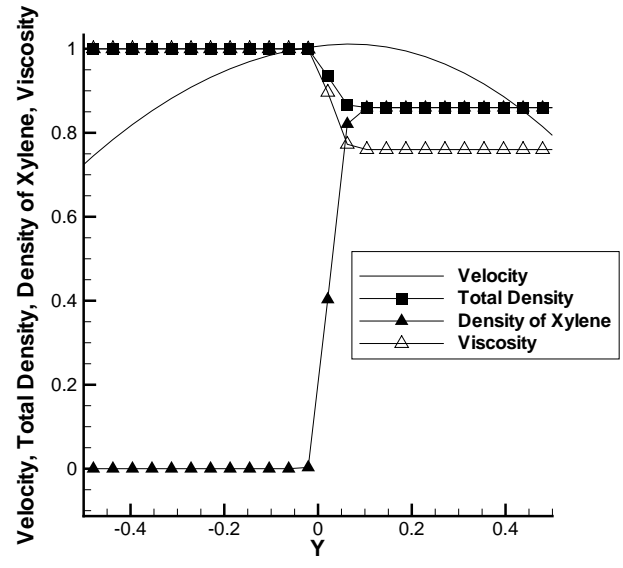

b)

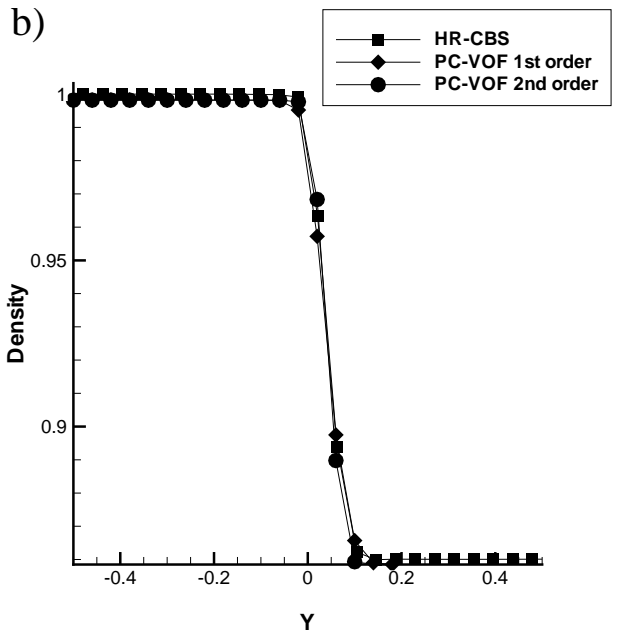

d)

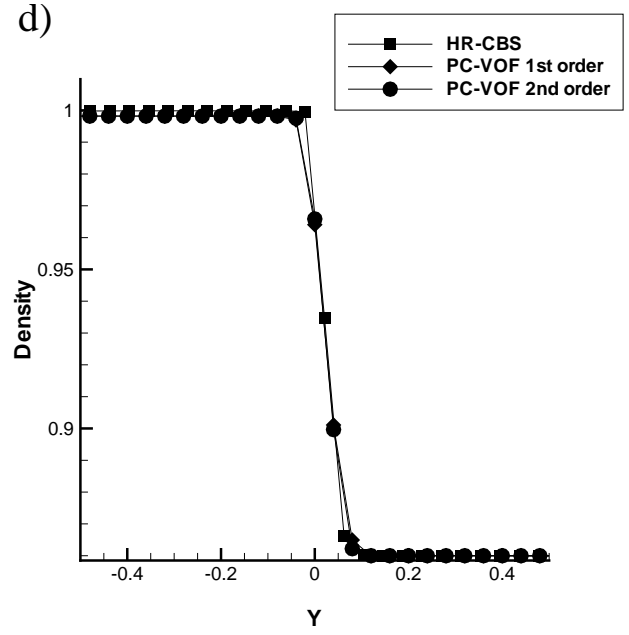

Figure 3: Solution for contact discontinuity: a) $\mu_{x w}=0.62$, HR-CBS model; b) $\mu_{x w}=0.62$ comparisons of different models; c) $\mu_{x w}=0.76$, HR-CBS model; b) $\mu_{x w}=0.76$, comparisons of different models

into account that the analytic value of the contact discontinuity position is approximated reasonably well by the middle-position in the density profile and that the actual magnitude of the interface displacement is small, we can re-map the grid as follows

$$
y_{i, j}^{\text {remapped }}=y_{i, j}\left(1-\operatorname{sign}\left(y_{i, j}\right) y_{i}^{\text {interface }}\right)+y_{i}^{\text {interface }}
$$

where $y_{i}^{\text {interface }}$ is the local position of the interface calculated by the middle-position in the density profile $(\rho=0.93)$. This leads to the grid line previously located at $y=0$ re-mapped onto the interpolated position of the interface.

Figure 4 shows the comparison of solution obtained with HR-CBS scheme on uniform and interface-fitted grids. The interface-fitted grid leads to slightly better resolution.

The mass flux correction (6) enables adjustment of the interface position. Figure 5 
a)

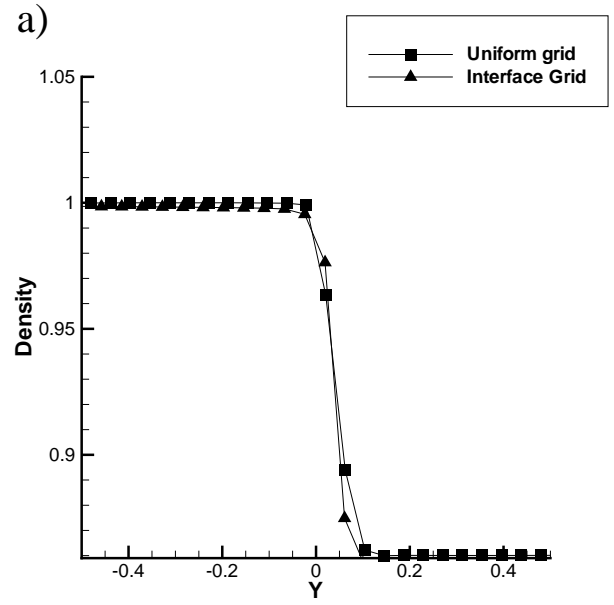

b)

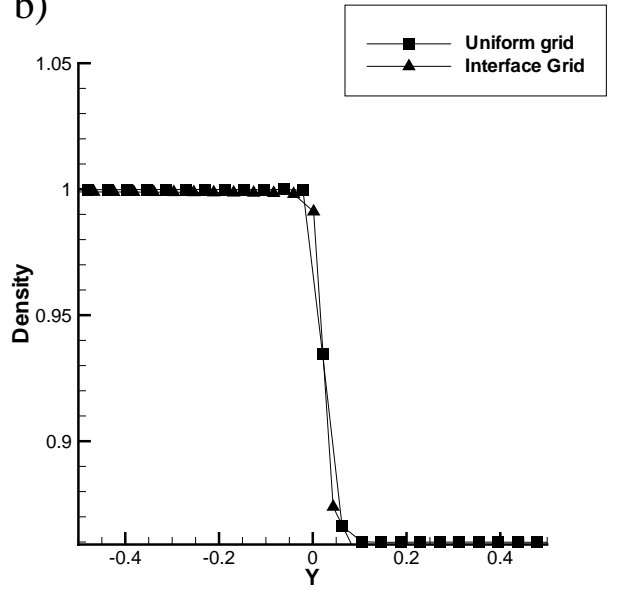

Figure 4: Comparison of contact discontinuity solutions on uniform and interface-fitted grids a) $\mu_{x w}=$ 0.62 b) $\mu_{x w}=0.76$.

\begin{tabular}{|c|c|c|c|c|}
\hline & PC VOF 1st & PC VOF 2nd & HR-CBS & Analytic \\
\hline \hline$\mu_{x w}=0.62$ & $0.0382(5 \%)$ & $0.0395(1.7 \%)$ & $0.04(0.5 \%)$ & 0.0402 \\
\hline$\mu_{x w}=0.76$ & $0.0216(5.7 \%)$ & $0.0217(5 \%)$ & $0.0236(3 \%)$ & 0.0229 \\
\hline
\end{tabular}

Table 2: Interface displacement in developed flow

shows the interface position with the volume flux corrected in the xylene inlet by $Q_{x w}=$ 1.130 and $Q_{x w}=1.072$ for $\mu_{x w}=0.62$ and $\mu_{x w}=0.76$, respectively. In both cases the middle-position of the density profile differs from 0 (centre of the channel) by $\sim 10^{-4}$.

\section{DISCUSSION}

Microfabrication related modelling of dual-fluid flow in microfluidics raises a number of interesting questions. The contact discontinuity resolution, which might be found adequate in other problems can lead to interface diffusion of the same order as the fabricated structures. The HR-CBS model yields better resolution of the contact discontinuity than the PC-VOF model, when the discontinuity is aligned to grid. However, for viscosity ratios resulting in interface displacement, both models yield similar results.

For liquids of different viscosity, the inlet volume flux can be adjusted using an analytic solution thus leading to centering of the contact discontinuity, which then becomes gridaligned and can be better resolved. Moreover, since the displacement of the interface is small, it is possible to fit the grid to the interface dynamically, which results in less dissipative solution for contact discontinuities corresponding to viscosity ratios close to one.

The readily available analytic solution for the fully developed flow makes the two- 
a)

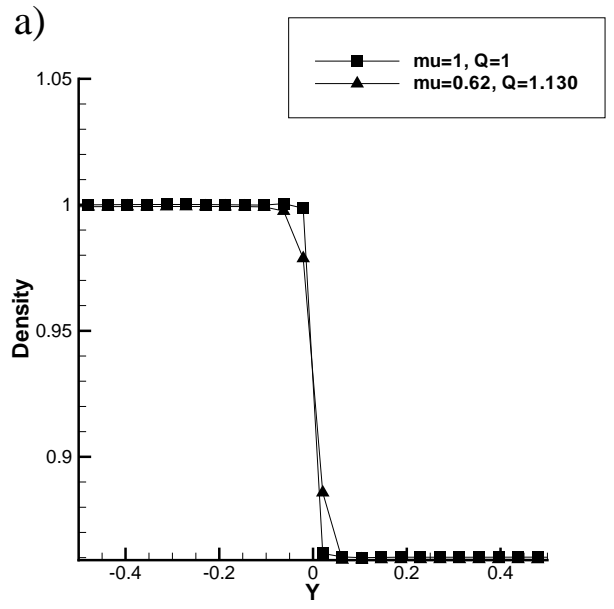

b)

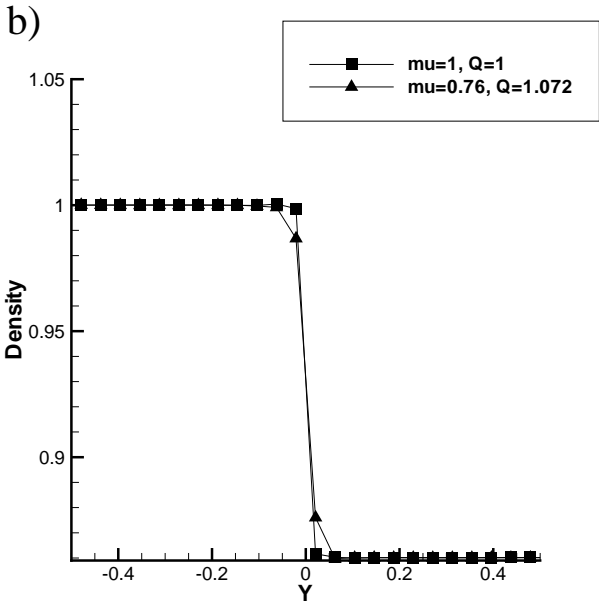

Figure 5: Discontinuity position as obtained when applying the mass flux correction: a) $\mu_{x w}=0.62$; b) $\mu_{x w}=0.76$

dimensional problem presented in this paper a suitable benchmark for modelling contact discontinuities in microfluidics.

\section{ACKNOWLEDGEMENTS}

The investigation was performed with the financial support of the Engineering and Physical Sciences Research Council (GR/S13668).

\section{References}

[1] P. J. A. Kenis, R. F. Ismagilov, and G. M. Whitesides. Microfabrication inside capillaries using multiphase laminar flow patterning. Science, 285:83-85, 1999.

[2] P. J. A. Kenis, R. F. Ismagilov, S. Takayama, G. M. Whitesides, S. Li, and H. S. White. Microfabrication inside microchannels using fluid flow. Accounts of Chemical Research, 33:841-847, 2000.

[3] H. Hisamoto, T. Saito, M. Tokeshi, A. Hibara, and T. Kitamori. Fast and high conversion phase-transfer synthesis exploiting the liquid-liquid interface formed in a microchannel. Chemical Communications, pages 2662-2663, 2001.

[4] H. Hisamoto, Y. Shimuzu, K. Uchiyama, M. Tokeshi, Y. Kikutani, A. Hibara, and T. Kitamori. Chemicofunctional membrane for integrated chemical process on a microchip. Analytical Chemistry, 75:350-354, 2003.

[5] B. Zhao, N.O.L. Viernes, J.S. Moore, and D.J. Beebe. Control and application of im- 
miscible liquids in microchannels. Journal of American Chemical Society, 124:52845285, 2002.

[6] J. Gargiuli, E. Shapiro, H. Gulhane, G. Nair, D. Drikakis, and P. Vadgama. Microfluidic systems for in situ formation of nylon 6,6 membranes. Journal of Membrane Science, in print, 2006.

[7] G. Nair, J. Garguili, N. R. Shiju, E. Shapiro, D Drikakis, and P Vadgama. In situ fabrication of crosslinked protein membranes using microfluidics. ChemBioChem, in print, 2006.

[8] E. Shapiro, D. Drikakis, J. Gargiuli, and P. Vadgama. Microfluidic cell optimization for polymer membrane fabrication. In Proceedings of the 4 th ASME International Conference on Nanochannels, Microchannels and Minichannels, June, Limerick, ICNMM2006-96221, 2006.

[9] S.G. Yiantsios and B.G. Higgins. Linear stability of plane poiseuille flow of two superposed fluids. Physics of Fluids, 31:3225-3238, 1988.

[10] S.G. Yiantsios and B.G. Higgins. Erratum: "linear stability of plane poiseuille flow of two superposed fluids". Physics of Fluids A, 1(5):897, 1989.

[11] E. Shapiro and D. Drikakis. Artificial compressibility, characteristics-based schemes for variable density, incompressible, multi-species flows. part i. derivation of different formulations and constant density limit. J. Comp. Phys., 210(2):584-607, 2005.

[12] E. Shapiro and D. Drikakis. Artificial compressibility, characteristics-based schemes for variable density, incompressible, multi-species flows. part ii. multigrid implementation and numerical tests. J. Comp. Phys., 210:608-631, 2005.

[13] D. Drikakis and W. Rider. High-Resolution Methods for Incompressible and LowSpeed Flows. Springer, 2004.

[14] R.Y. Chen. Flow in the entrance region at low reynolds numbers. Transactions of ASME, Journal of Fluids Engineering, 95:153-158, 1973.

[15] R.M. Sadri and J.M. FLoryan. Entry flow in a channel. Computers E Fluids, 31:133157, 2002.

[16] A. J. Chorin. A numerical method for solving incompressible viscous flow problems. J. Comput. Phys., 2, 1967. 